\title{
Post-Myocardial Infarction Exercise Training Induces Angiogenesis in the heart
}

\author{
Bryan S Wilson, Michael A Garza and John Q Zhang* \\ Laboratory of Cardiovascular Research, University of Texas at San Antonio, San Antonio, TX 78249, USA
}

Received: December 29, 2017; Accepted: January 24, 2018; Published: January 30, 2018

*Corresponding author: John Q Zhang, Professor of Exercise Physiology, Laboratory of Cardiovascular Research, University of Texas at San Antonio, One UTSA Circle, San Antonio, TX 78249, USA, Tel: 1-210-458-7390; Fax: 1-210-458-5873; Email: john.zhang@utsa.edu

\begin{abstract}
Cardiac angiogenesis after myocardial infarction (MI) is critical to the development of compensatory hypertrophy in the viable myocardium; however, compelling evidence suggests that post-MI cardiac angiogenesis is inadequate, and may promote the transition from adaptive cardiac hypertrophy to left ventricular (LV) dilation and dysfunction. Exercise, through increased vascular shear stress, potentiates a powerful antigenic stimulus. We investigated whether exercise could promote cardiac angiogenesis in post-MI rats. MI was surgically induced on 7-wk-old Sprague-Dawley rats by ligation of the coronary artery. The survivors were assigned to 3 groups ( $\mathrm{n}=10 /$ group): Sham (no MI, no exercise), MISed (MI, no exercise), and MIEx (MI + exercise). Treadmill exercise training began 1-wk post-MI and lasted for 8-wks. Cardiac tissue was harvested at the end of the experiment and capillary density was assessed using CD31 staining. Our results indicated that left ventricular (LV) capillary density (counts $/ \mathrm{mm}^{2}$ ) of Sham and MIEx groups were significantly higher compared to the MISed group $\left(1280 \pm 66\right.$ counts $/ \mathrm{mm}^{2}, 950 \pm$ $47,610 \pm 44$, respectively, $\mathrm{p}<0.05$ ). In the septum, capillary density was also higher in the MIEx group compared to MISed (1220 \pm 54 vs. $890 \pm 43$ counts $/ \mathrm{mm}^{2}$ ). Conclusion: Our data indicate that postMI exercise training at a moderate intensity significantly increases cardiac angiogenesis, which in turn may attribute to the improvement in morbidity and mortality produced by exercise training in patients with MI.
\end{abstract}

Keywords: myocardial infarction; exercise training; angiogenesis; animal model;

\section{Introduction}

Cardiovascular disease remains the leading cause of morbidity and premature mortality despite advances in medical therapy, such as percutaneous coronary interventions and surgical revascularization [14]. After myocardial infarction (MI), the adequate growth of new capillaries and arterioles, or angiogenesis, represents a critical process in the development of compensatory hypertrophy in the remaining non-infarcted myocardium [2]. Although compensatory angiogenesis can be observed in both the ischemic and infarcted heart, previous studies have demonstrated that angiogenesis may be inadequate[12]; in fact, recent evidence suggests that impaired angiogenesis may lead to maladaptive left ventricular (LV) remodeling, promoting the transition from adaptive cardiac hypertrophy to $\mathrm{LV}$ dilation and dysfunction[18].
Exercise, through increased vascular shear stress, potentiates a powerful angiogenic stimulus [5]. In this regard, a number of studies clearly demonstrate that exercise activates vascular endothelial growth factor (VEGF) dependent antigenic pathways $[17,3,10,11]$ which represent critical molecular mechanisms by which exercise triggers angiogenesis [11]. Previous studies by our group and others have demonstrated that exercise training positively influences cardiac function and attenuates myocardial remodeling in rats with MI or congestive heart failure $[11,7$, $1,19] \mathrm{n}$ addition, exercise-induced up regulation of VEGF in patients with heart failure has also been documented [9]. To date, however, few studies have examined the effects of exerciseinduced angiogenesis in the infarcted myocardium; therefore, the purpose of this study was to investigate the effects of exercise training on cardiac angiogenesis in post-MI rats.

\section{Methods and Materials}

\section{Animal Preparation}

TSeven-week-old (185-200 g) male Sprague-Dawley rats (Harlan, Indianapolis, IN) were treated in accordance with National Institutes of Health Guide for the Care and Use of Laboratory Animals (ILAR XXXX), and study protocols were approved by the Institutional Animal Care and Use Committee of the University of Texas at San Antonio. To ensure the rats were accustomed to running, they were trained on a rodent treadmill at 10 to $16 \mathrm{~m} / \mathrm{min}, 5 \mathrm{~min} /$ day for one week prior to surgery. MI was surgically induced by ligation of the left anterior descending coronary artery as described previously [25].

One week after surgery, the surviving rats were matched with cardiac function (Fractional Shortening) determined by echocardiography measurement and randomly assigned to three experimental groups ( $\mathrm{n}=10 /$ group): a sham-operated control (Sham), a sedentary group with MI (MI-Sed) and an exercise group with MI (MI-Ex). The MI-Ex group started exercising one week post-MI using a motorized rodent treadmill, while the Sham and MI-Sed groups remained sedentary throughout the entire experiment. To allow gradual adaptation to exercise stress, training was initiated at $10 \mathrm{~m} / \mathrm{min} ; 5^{\circ}$ incline for 10 min per session. The speed and duration were gradually increased to 16 $\mathrm{m} / \mathrm{min}$ and 50 min per session (including a 5 -min warm-up at $10 \mathrm{~m} / \mathrm{min}$ ) and maintained constant throughout the experiment. 
The exercise training was performed 5 days per week for 8 weeks. The determination of treadmill speed and exercise duration was based on the previous studies [20, 4, 25]. This exercise regimen was well tolerated by rats with MI. There were no mortalities during the 8 weeks of exercise training.

\section{Cardiac Tissue Collection}

Rats were anesthetized forty-eight hours after the last exercise session. The hearts were quickly harvested and rinsed in cold saline. The myocardial tissue of the non-infarcted left ventricle (LV) was collected and immediately frozen in is pentane with dry ice. Tissues and serum were stored at $-80^{\circ} \mathrm{C}$ until use.

\section{Infarct size determination}

Six-micrometer thick sections of the heart were cut and stained with Masson's trichrome. Infarct size was calculated by dividing the sum of the plan metered endocardial and epicardial circumferences of the infarcted area by the sum of the total epicardial and endocardial circumferences of the LV [25]. Total epicardial and endocardial lengths occupied by the infarct as identified by Masson's trichrome staining was measured using Image Pro Plus program (Media Cybernetics, Silver Spring, MD).

\section{Capillary Density}

In brief, the protocol for determining capillary density was a two-stage immunohistochemical process involving marking for CD-31 and light microscopy quantification. After fixation, 6um transverse cross-sections were incubated at $4^{\circ} \mathrm{C}$ overnight with primary antibody (1:20 dilution) anti-CD-31 antibody (BD \#550300) in a humid chamber. After rinsing off the primary anti-body, a secondary anti-body enhancement kit (DAKO \#K0609) with a biotynilated link and streptavidin-HRP was applied according to manufactures' instructions. Stains were then developed via diaminobenzidine (DAB) table sets (Sigma D-4293). After dehydration and mounting of the slides, images were obtained using an inverted Olympus Q-Fire side mounted digital camera. The capillary density was determined by counting capillaries from 3 equally spaced images of the LV wall (remote area of the LV for the MISed and MIEx groups), 3 equally spaced images of the intraventricular septum, and 3 equally spaced images of the right ventricle of the myocardium cross-section using Image Pro Plus software (ver. 5, Media Cybernetics). Using a point to count technique with Photoshop (Adobe ver. 10), results was expressed as counts $/ \mathrm{mm}^{2}$ of myocardium. Capillaries were defined as $<10 \mathrm{um}$ in diameter. A stage micrometer was used to standardize the microscope, the imaging software, and the measurement area within the counting software as adapted from previous studies.

\section{Statistical Analysis}

Values are expressed as mean \pm standard error of mean (SEM). The group means were compared with one-way analysis of variance (ANOVA) and significant $\mathrm{F}$ ratios was followed by Student-Newman-Keuls post hoc comparisons. A P value of less than 0.05 was considered statistically significant.

\section{Results}

\section{General Characteristics}

Following ligation, there was a $\sim 50 \%$ mortality rate with all the deaths occurring after the procedure. The exercise protocol was well tolerated and no deaths were observed throughout the treatment period of the study. The general characteristics for the study population are summarized in table 1 . Infarct percentages among the MIEx and MISed groups were comparable. Total heart weight was significantly higher in the MIEx and MISed groups compared to the Sham group $(1.46 \pm 0.04 \mathrm{~g}, 1.42 \pm 0.05$ vs. $1.14 \pm$ 0.04 , respectively, $\mathrm{p}<0.05$ ). Similarly, heart to body weight ratios were also significantly higher in the MIEx and MISed groups compared to the Sham group $(3.65 \pm 0.07 \mathrm{~g}, 3.58 \pm 0.2$ vs. 3.24 \pm 0.1 )

Unsuccessful PCI procedure related complications included

\begin{tabular}{|l|c|c|c|c|}
\hline \multicolumn{5}{|l|}{ Table 1: General Characteristics } \\
\hline & $\begin{array}{c}\text { Infarct } \\
\text { Size (\%) }\end{array}$ & BW (g) & Ht Wt (g) & $\begin{array}{c}\text { Ht Wt (g)/ } \\
\text { BW (kg) }\end{array}$ \\
\hline Sham & \pm & $352 \pm 3.4^{*}$ & $1.14 \pm 0.04^{*}$ & $3.24 \pm 0.1^{*}$ \\
\hline MI-Ex & $37.5 \pm 2.6$ & $400 \pm 9.3$ & $1.46 \pm 0.04$ & $3.65 \pm 0.07$ \\
\hline MI-Sed & $33.7 \pm 2.4$ & $398 \pm 7.8$ & $1.42 \pm 0.05$ & $3.58 \pm 0.2$ \\
\hline
\end{tabular}

Values are expressed as mean $+\mathrm{SEM} .{ }^{*} \mathrm{P}<0.05$ Sham versus the MI-Ex and MI-Sed groups.

\section{Capillary Density}

Figure 4 shows the representative images of the immunohistochemical CD-31 staining of LV capillaries, which were visualized using a light microscope at $20 \mathrm{x}$ magnifications and appear brown in color. Our results indicated that capillary density in the septum (Figure 1) was significantly higher in the MIEx group compared to MISed (1220 \pm 54 vs. $890 \pm 43$ counts/ $\mathrm{mm} 2, \mathrm{p}<0.05$ ). Furthermore, LV capillary density (Figure 3 ) of Sham and MIEx groups were significantly higher compared to the MISed group $(1280 \pm 66$ counts/mm2, $950 \pm 47,610 \pm 44$, respectively, $\mathrm{p}<0.05$ ), thus indicating that exercise significantly enhanced cardiac angiogenesis in the septum and LV nine weeks after MI. Such effects were not observed in the right ventricle (Figure 2).

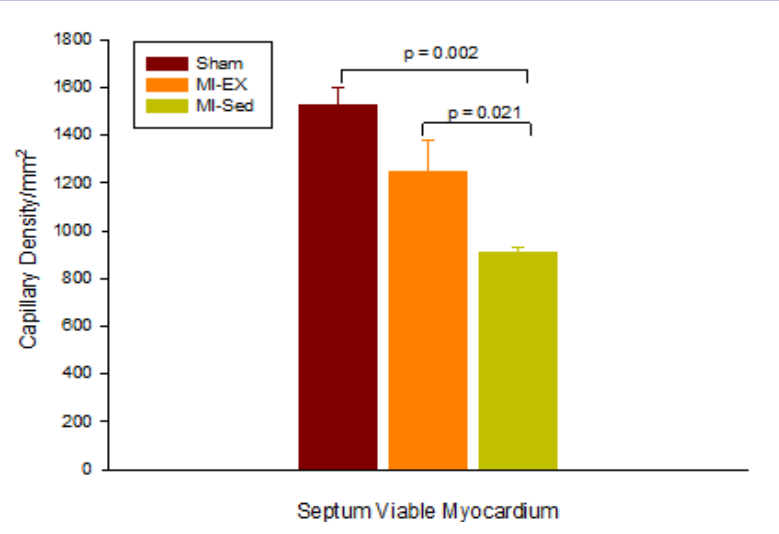

Figure 1: Immunohistochemical staining (CD 31) results of septum capillary density. 


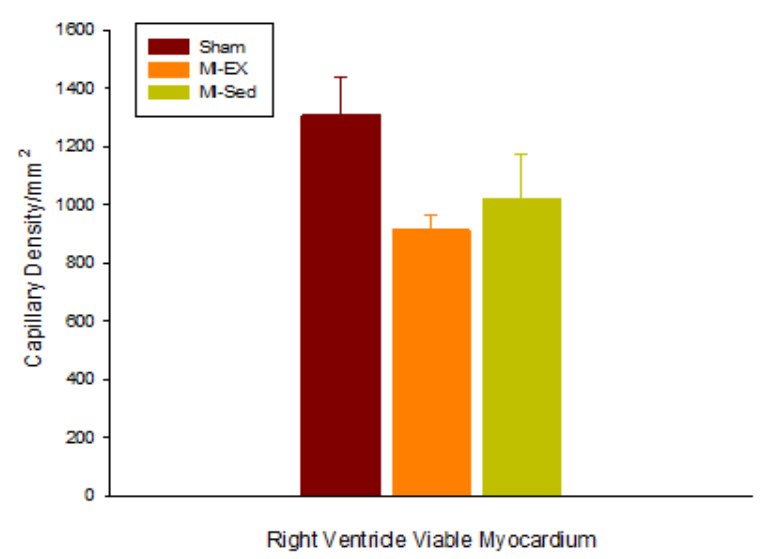

Figure 2: Immunohistochemical (CD 31) results of right ventricle capillary density

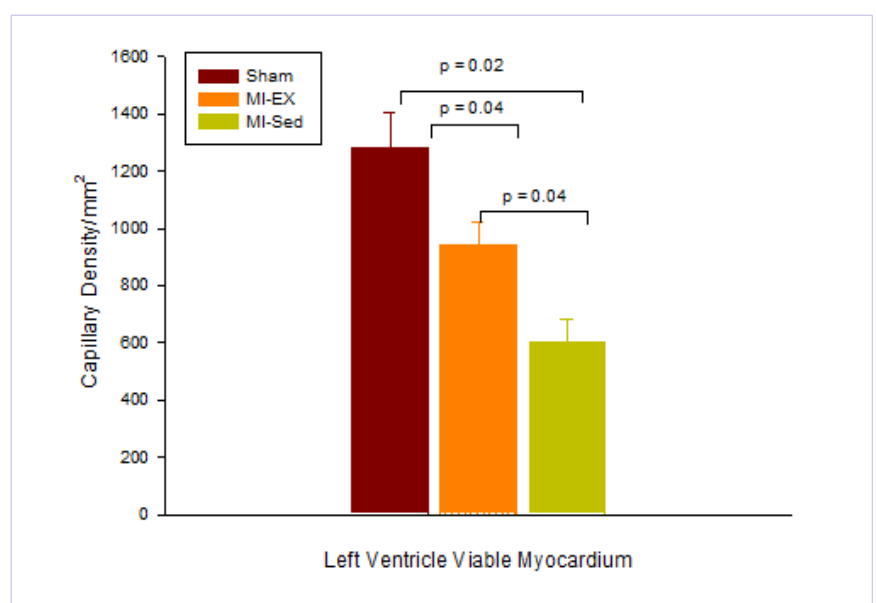

Figure 3: Immunohistochemical (CD 31) results of left ventricle capillary density
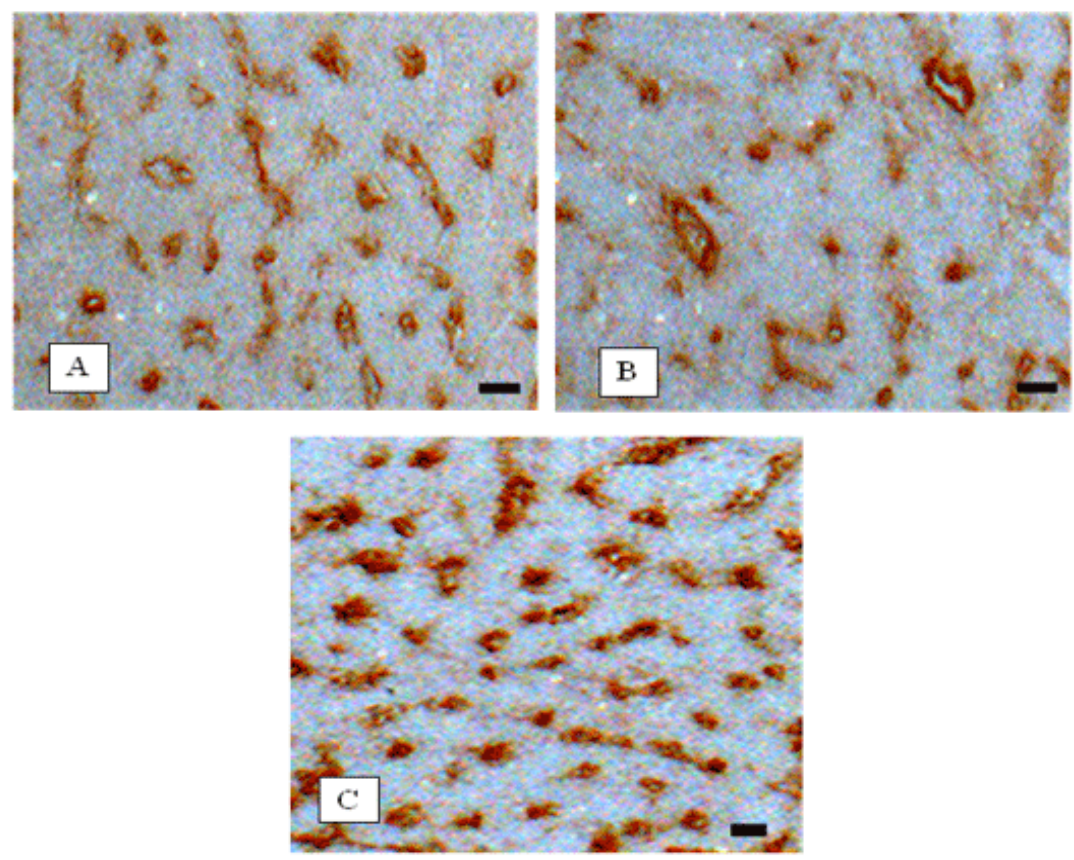

Figure 4: Representative image of the immunohistochemical CD 31 staining of LV capillaries, which appear brown in color: (A) MI-Ex (B) MI-Sed, and (C) Sham. Capillaries are visualized using a light microscope at 20x magnification. The bar in the photomicrographs indicates $10 \mu \mathrm{m}$.

\section{Discussion}

In a rat model of severe MI, we demonstrated that exercise training significantly increases capillary density in both the $\mathrm{LV}$ and septum of the viable myocardium. Exercise-induced angiogenesis such as that observed in our study may enhance myocardial blood perfusion and improve cardiac functioning in the infarcted heart. These data provide further insights into the mechanisms underlying the improvement in morbidity and mortality produced by exercise training in patients with MI.

Our results confirm previous evidence showing that low to moderate intensity treadmill exercise started late after MI beneficially impacts cardiac function and attenuates adverse remodeling $[16,23]$. Contrarily, training programs consisting of higher exercise intensities, and started early after MI have reported detrimental effects on LV geometry and mortality in rats $[13,6]$. The use of endurance swimming, which elicits higher mental and hemodynamic stresses compared with treadmill exercise, in addition to increased training frequency $(90 \mathrm{~min} / \mathrm{d}$, $6 \mathrm{~d} / \mathrm{wk}$ ) strongly differentiates these previous exercise protocols from that used in the present investigation, and may account for the negative effects of exercise in HF animals observed by these authors [6]. 
In the present study, our results illustrated that MI-Ex capillary density in the septum and left ventricle was 1.4 -fold higher (Figure 1) and 1.6-fold higher (Figure 3) than in MI-Sed $(1220 \pm 54$ vs. $890 \pm 43$, and $950 \pm 47$ vs. $610 \pm 44)$, suggesting that exercise promotes capillary growth in non-infarcted areas of severely decompensate hearts. Similarly, a study conducted by Leosco et al. reported that exercise induced a significant increase of capillary density in lateral border and remote zones of LV in HFEX hearts, but not in the LV anterior wall, which was largely involved in the infarcted area [15]. Although the pro-antigenic effect of exercise has previously been demonstrated in healthy swine hearts [22], data from the present study, in addition to the work of Leosco and colleagues, represents the only literature on exercise-dependent enhanced cardiac angiogenesis after MI. Our results showed that there was no significant difference in the ratio of heart weight to body weight regardless of the higher capillary density observed in the exercised trained LV. Although angiogenesis is contributed to hypertrophy of the heart [2] Post-MI cardiac hypertrophy is mainly caused by angiotensin II mediated cell proliferation [8]. Our previous studies [21, 24] demonstrated that post-MI exercise training attenuated both plasma and cardiac tissue angiotensin II. This may explain why the heart weight to body weight ratio in the MI-EX group did not differ from their sedentary counterpart.

In conclusion, moderate intensity exercise training significantly increases capillary density in both the LV and septum of the viable myocardium. In this regard, exercise induced angiogenesis has the potential to impact numerous clinical conditions involving the ischemic and infarcted myocardium.

\section{Acknowledgement}

This study was supported in part by a grant from the National Heart, Lung, and Blood Institute (R01-HL074273).

\section{References}

1. Alhaddad IA, Hakim Ishrat, Siddiqi Faizi, Lagen back Edward, Mallavarapu Christopher, Nethala, Venkatram et al. Early exercise after experimental myocardial infarction: effect on left ventricular remodeling. Coron Artery Dis. 1998;9(6): 319-327.

2. Anversa P, Beghi C, Kikkawa Y, Olivetti G. Myocardial infarction in rats. Infarct size, myocyte hypertrophy, and capillary growth. Circ Res. 1986;58(1):26-37.

3. Bloor CM. Angiogenesis during exercise and training. Angiogenesis. 2005;8(3):263-271.

4. Carlson CL, WW Winder. Liver AMP-activated protein kinase and acetyl-CoA carboxylase during and after exercise. J Appl Physiol. 1985;86(2):669-674.

5. Egginton S. Invited review: activity-induced angiogenesis. Pflugers Arch.2009;457(5): 963-977.

6. Gaudron P, Hu K, Schamberger R, Budin M, Walter B, Ertl G. Effect of endurance training early or late after coronary artery occlusion on left ventricular remodeling, hemodynamics, and survival in rats with chronic transmural myocardial infarction. Circulation. 1994;89(1):402-412.
7. Giannuzzi P, Temporelli PL, Corrà U, Gattone M, Giordano A, Tavazzi L. Attenuation of unfavorable remodeling by exercise training in postinfarction patients with left ventricular dysfunction: results of the Exercise in Left Ventricular Dysfunction (ELVD) trial. Circulation. 1997;96(6):1790-1797 .

8. Gray MO, Long CS, Kalinyak JE, Li HT, Karliner JS. Angiotensin II stimulates cardiac myocyte hypertrophy via paracrine release of TGF-beta 1 and endothelin-1 from fibroblasts. Cardiovasc Res. 1998;40(2):352-363.

9. Gustafsson, TBodin K, Sylvén C, Gordon A, Tyni-Lenné R, Jansson E. Increased expression of VEGF following exercise training in patients with heart failure. Eur J Clin Invest. 2001;31(4):362-366.

10. Iemitsu M, Maeda S, Jesmin S, Otsuki T, Miyauchi T. Exercise training improves aging-induced downregulation of VEGF angiogenic signaling cascade in hearts. Am J Physiol Heart Circ Physiol. 2006;291(3): H1290-1298.

11. Jugdutt BI, Bogdan L Michorowski, C Tissa Kappagoda. Exercise training after anterior $\mathrm{Q}$ wave myocardial infarction: importance of regional left ventricular function and topography. J Am Coll Cardiol. 1988;12(2):362-372. DOI: 10.1016/0735-1097(88)90407-X

12. Karam R, Healy BP, Wicker P. Coronary reserve is depressed in postmyocardial infarction reactive cardiac hypertrophy. Circulation. 1990;81(1):238-246

13. Kloner RA, JA Kloner. The effect of early exercise on myocardial infarct scar formation. Am Heart J. 1983;106(5-1):1009-1013. Doi

14. Krichavsky MZ, DW Losordo. Prevention and recovery of hibernating myocardium by micro vascular repair. Circulation. 2011;124(9):9981000. Doi.org/10.1161/CIRCULATIONAHA.111.047746

15. Leosco, D Rengo G, Iaccarino G, Golino L, Marchese M, Fortunato F. Exercise promotes angiogenesis and improves beta-adrenergic receptor signalling in the post-ischaemic failing rat heart. Cardiovasc Res. 2008;78(2):385-394.

16. Musch TI, Moore RL, Leathers DJ, Bruno A, Zelis R. Endurance training in rats with chronic heart failure induced by myocardial infarction. Circulation. 1986;74(2):431-441.

17. Prior BM, Yang HT, Terjung RL. What makes vessels grow with exercise training?. J Appl Physiol.2004;97(3):1119-1128

18. Shiojima I, Kaori Sato, Yasuhiro Izumiya, Stephan Schiekofer, Masahiro Ito,Ronglih Liao, et al. Disruption of coordinated cardiac hypertrophy and angiogenesis contributes to the transition to heart failure." J Clin Invest. 2005;115(8):2108-2118. Doi:10.1172/ JCI24682

19. Sysa-Shah, P Yi Xu , Xin Guo, Frances Belmonte, Byunghak Kang, Djahida Bedja. Cardiac-Specific Over-Expression of Epidermal Growth Factor Receptor 2 (ErbB2) Induces Pro-Survival Pathways and Hypertrophic Cardiomyopathy in Mice." PLoS One. 2012;7(8):e42805.

20. Veras-Silva AS, Mattos KC, Gava NS, Brum PC, Negrão CE, Krieger EM. Low-intensity exercise training decreases cardiac output and hypertension in spontaneously hypertensive rats. Am J Physiol. 
1997;273(6 Pt 2): H2627-2631.

21. Wan W, Anthony S PowersBS, Ji Li, John Q Zhang, Lisa Ji, John M.Erikson. Effect of post-myocardial infarction exercise training on the renin-angiotensin-aldosterone system and cardiac function. Am J Med Sci. 2007;334(4):265-273. Doi.org/10.1097

22. White FC, Bloor CM, McKirnan MD, Carroll SM. Exercise training in swine promotes growth of arteriolar bed and capillary angiogenesis in heart. J Appl Physiol. 1998;85(3):1160-1168.

23. Wisloff U, Jan P Loennechen, SusanCurrie, Godfrey L Smith, Øyvind Ellingsen. Aerobic exercise reduces cardiomyocyte hypertrophy and increases contractility, Ca2+ sensitivity and SERCA-2 in rat after myocardial infarction. Cardiovasc Res. 2002;54(1):162-174. doi. org/10.1016/S0008-6363(01)00565-X

24. Xiaohua Xu, Wenhan Wan, Lisa Ji, Shunhua Lao, Anthony S Powers,
Weiyan Zhao, John M Erikson. Exercise training combined with angiotensin II receptor blockade limits post-infarct ventricular remodelling in rats. Cardiovasc Res. 2008;78(3);523-532. Doi. org/10.1093/cvr/cvn028

25. XiaohuaXu, Wenhan Wan, Anthony S Powers, JiLi, Lisa L Ji, ShunhuaLao et al. Effects of exercise training on cardiac function and myocardial remodeling in post myocardial infarction rats. J Mol Cell Cardiol. 2008;44(1):114-122. Doi.org/10.1016/j.yjmcc.2007.10.004 